\title{
Liquid biopsy in cancer using the Z-scan technique: a new approach to discover biomarkers in cancer
}

\author{
Fernando Luiz Affonso Fonseca*,1,2 (D), Glaucia Luciano da Veiga1 (iD), Beatriz da Costa \\ Aguiar Alves $^{1}$ (i) \& Sarah Isabel Pinto Monteiro do Nascimento Alves ${ }^{2}$ (D) \\ ${ }^{1}$ Clinical Analysis Laboratory, Centro Universitário Saúde ABC, Santo André 06090-650, Brazil \\ ${ }^{2}$ Instituto de Ciências Ambientais, Químicas e Farmacêuticas (ICAQF), Universidade Federal de São Paulo - UNIFESP, Diadema \\ 09972-270, Brazil \\ *Author for correspondence: Tel.: +55 114993 5488; profferfonseca@gmail.com
}

\begin{abstract}
Aim: Biomarkers have been broadly studied as a tool for the diagnosis and prognosis for different types of cancer. Z-scan is a kind of measurement technique that generates a nonlinear refractive index $\left(\mathrm{n}_{2}\right)$. Today, Z-scan has been used in oncology to discriminate between solid tumors and to identify tumor circulating cell-free DNA in liquid samples. Materials \& methods: According to the inclusion and exclusion criteria defined in this review, 35 articles were selected. Conclusion: The use of this technique for this kind of measurement will allow for a rapid and precise diagnosis of different types of tumor and may lead to better therapeutic approaches.

Lay abstract: Biomarkers are molecules, proteic or not, found in different body fluids or tissues. The study of these molecules makes it possible to diagnose several diseases in their initial stages, being fundamental for the control of pathologies. Z-scan is a nonlinear measurement technique capable of detecting some of these molecules. Its use has been considered for cancer diagnosis. This review provides pertinent information for better understanding this method and its importance for diagnostic medicine.
\end{abstract}

First draft submitted: 30 March 2020; Accepted for publication: 16 September 2020; Published online: 20 October 2020

Keywords: biomarker $\bullet$ cancer $\bullet$ diagnostic $\bullet$ liquid biopsy $\bullet$ Z-scan

\section{Biomarkers}

A biomarker is an analytically measurable and quantifiable molecule used in the diagnostic and prognostic evaluation of many pathologies aiming to extract pertinent information about health conditions or pathological processes. A reliable biomarker has molecular, biochemical, histological and radiological characteristics [1]. According to the definition of the National Institutes of Health (MD, USA), a biomarker has to be able to indicate not only a normal or pathological biological process, but also the individual's response to a pharmacological treatment and/or a therapeutic intervention [2]. In sum, a biomarker has to be able to provide a set of data that will guide the medical approach.

Biomarkers are classified according to their clinical purpose. This classification has been strategically divided to facilitate its applicability [3]: those which are able to confirm the presence of a pathology are called diagnostic biomarkers; those that monitor the progression or remission of a disease are denominated prognostic biomarkers. There are also the predictive biomarkers, which reflect a patient's response to a therapy. Moreover, adverse reaction, safety and dose-response biomarkers are used, especially upon the administration of a specific drug $[1,4,5]$.

\section{Classification of biomarkers}

In order to consider a biomarker evaluation trustworthy (diagnosis, prognosis or therapeutic follow-up), it has to be as safe and effective as possible. Analytical instruments can measure specificity and sensitivity of candidates to biomarkers and ensure a high positive predictive value or exclude the possibility of false-negative results [6]. A 
biomarker is considered to have good sensitivity when it can detect the disease whenever it is really there (true positive). Specificity refers to the acknowledgement of the true absence of the disease. This evaluation is made through the analysis of the receiver operating characteristic curve, which is the graphic representation that illustrates the performance of a biomarker according to a binary combination and changes in the discrimination threshold. This evaluation not only selects ideal models of analysis, but also disregards unsuitable models, a fact that makes the receiver operating characteristic curve be considered the gold standard in studies on biomarkers $[7,8]$.

The classification of biomarkers is based on indicators of traces of the disease (genetic tests, family screening, etc), as well as the stage of the disease or the progression rate (preclinical phase and confirmation of the diagnosis). Antecedent biomarkers analyze the risk for the development of the disease in a population with risk factors whereas prognostic biomarkers provide the clinical basis to evaluate the response to the treatment. Furthermore, all biomarkers should be able to identify the risk for the development of a specific disease, screen subclinical diseases, recognize the disease at diagnosis, determine the disease staging and predict the course of the disease or the response to treatment [9].

\section{The nonlinear optical method (Z-scan) \& its applications}

In the past few years, the nonlinear optical technique called $Z$-scan $[10,11]$ has been applied in many areas, including health. The technique in well established in health processes, especially for the evaluation of human lipoprotein oxidative stress $[12,13]$ or for the quantification of levels of total cholesterol, triglycerides, protein, albumin and glucose in blood [14,15]. It has also been used to relate atherosclerosis to periodontal disease [16].

The Z-scan technique allows for the study of the nonlinear optical properties of many materials due to the fact it enables the determination of not only the nonlinear refractive index $\left(\mathrm{n}_{2}\right)$, but also the nonlinear absorption $(\beta)$ of the material through a characteristic signal emitted from the material in the presence of an intense light source. The presence of this signal may be related to changes in the morphology in part of the material or to variations in size and composition.

The main phenomenon in this technique is the variation in the refractive index of the medium caused by the intensity (I) of the light beam that falls on the sample. This process is represented according to the formula below:

$$
n=n_{0}+\Delta n
$$

where $n_{0}$ is the linear part and $\Delta n$ is the variation of the refractive index of the sample. The variation of the refractive index $(\Delta n)$ in the sample may be of electronic origin $\left(\Delta n=n_{2} \cdot I\right)$ or of thermal origin, caused by the temperature variations $(\mathrm{T})\left(\Delta n=n_{2} \cdot \Delta T\right)$ and concentration $(\Delta \phi)\left(\Delta n=n_{2} \cdot \Delta \phi\right)$. In other words, the refractive index of a medium depends on many different factors $[13,17,18]$.

This technique consists of moving a sample of the studied material along the transmission direction ( $\mathrm{Z}$ axis) of a focused Gaussian laser beam closer to the focal region $(z=0)$ (Figure 1). In this technique, the transmittance of a laser beam upon hitting the sample at a far-field observation is considered.

A photodetector is placed behind an iris and both are located at a point that is distant from the focal region. The photodetector will measure the intensity variation of the beam emitted by the sample $(\mathrm{I}(\mathrm{z}))$ according to the position of the sample. The resultant effect on the sample during the Z-scan process is focusing or defocusing, depending on the signal of the nonlinear refractive index.

Whenever the nonlinear effect results from a thermal origin, the sample will have an illuminated and heated area when interacting with the laser beam due to the partial absorption of the beam energy. The sample will lose heat to the surroundings once it is no longer illuminated. Since the laser beam has a Gaussian profile, the temperature gradient $\Delta T$ will be responsible for the variation of the refraction index $\left(n=n_{0}+n_{2} \cdot \Delta T\right)$.

Depending on the origin of the nonlinear effect, there are many theoretical models for the analysis of the results, such as the Sheik-Bahae model, the aberrant thermal lens model among others $[10,11,14,17,18]$.

In order to discover new biomarkers, two complementary approaches are adopted: the deductive method and the inductive strategy. Besides, the unbiased approach is applied to study a vast number of molecules through specific and modern methodologies, which characterizes a molecular signature for a specific pathology [6]. For this reason, nonlinear detection methodologies may be among the most modern technologies regarding to the fine and precise detection of molecules. 
Figure 1. Z-Scan experimental setup.

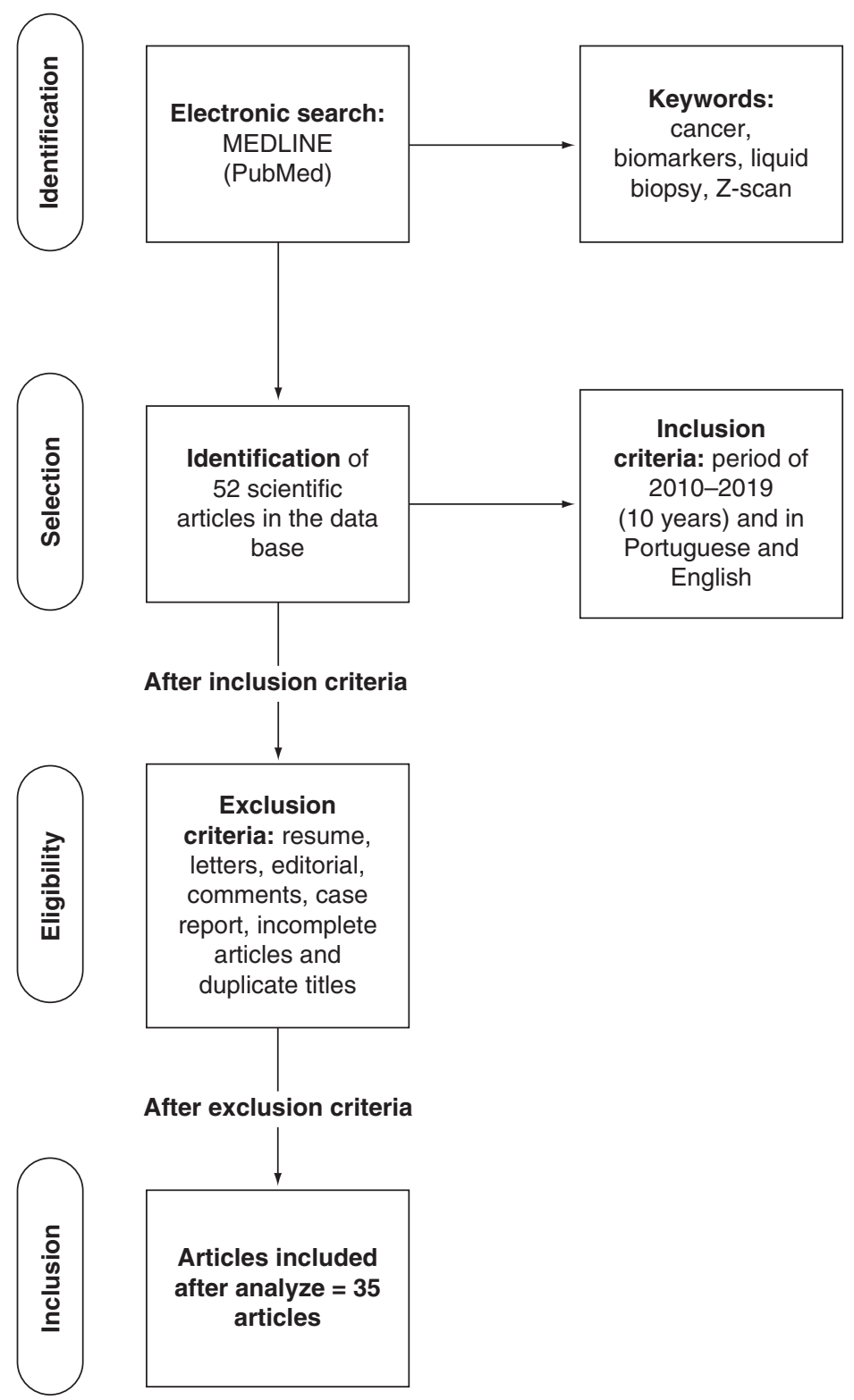

\section{Materials \& method}

A total of 52 articles searches were selected in database MEDLINE (PubMed) using the keywords: 'cancer', 'biomarkers', 'liquid biopsy' and 'Z-scan' combined with the Boolean 'AND' to obtain the outcomes of interest for the years 2010-2019. All searches in databases were done by two researchers following the protocol to ensure both obtained the same results from all the databases and they are trustable.

The inclusion criteria: specific approach to innovative methodologies of minimal invasion to cancer patients and Z-scan method in Portuguese and English for the last 10 years. Exclusion criteria: resume, letters, editorials, comments, case reports, incomplete articles and duplicate of titles. Therefore, at the end, 35 articles remained that fit within the inclusion and exclusion criteria (Figure 1).

\section{Results \& conclusion}

\section{Liquid biopsy in cancer}

There has been an increase in number of studies searching for new candidates for biomarkers in the clinical and diagnostic areas. These studies have cutting-edge technology on their side, such as combinatorial chemistry methodology, mass spectrometry, high-performance screening, proteomics and DNA microarrays. Liquid biopsy 
is a minimally invasive technique with high diagnostic and prognostic potential that can develop an individual molecular profile [19]. The term liquid biopsy was introduced in biochemical studies as a technology for the analysis of circulating tumor cells, factors derived from tumor cells or circulating tumor DNA (ct-DNA). This technique provides the detection and characterization of minimal residual diseases a result of the presence of disseminated tumor cells, which are sometimes distant from the primary lesion. The advantage of the use of this technique is the possibility to identify the presence of these tumor cells in patients who lack clinical or radiological signs of metastasis, thus contributing to the introduction of an early treatment and the possibility to revert the condition or improve the patient's prognosis [20]. Nowadays, recent attempts to combine the technology of liquid biopsy and physics techniques of molecule detection, like Z-scan, are believed to be a watershed in the early diagnosis of diseases like cancer.

In oncology, biopsy of the affected tissue has been the gold standard for the diagnosis, providing not only histopathological but also genetic information about the lesion [21]. Nevertheless, the collection of tissue biopsy specimens and their processing for pathological analyses involve challenges as it is an invasive surgical procedure with inherent risks, and the biological material obtained may not be enough for genomic investigation. Additionally, the genomic evaluation is usually done on different fragments of the tissue, due to the fact that tumors are heterogeneous. The collected material is generally preserved in paraffin blocks, which may lead to alterations in the genetic material [22].

Liquid biopsies have been in the spotlight for some time given the fact they comprise the analysis of biomarkers for the monitoring of diseases in biological matrices obtained through a noninvasive or minimally invasive technique. Peripheral blood is mostly used because cell-free DNA (cf-DNA) or circulating cell-free plasma DNA (ccf-pDNA) can be found in it [20]. Both normal and tumor cells originate cf-DNA, and in tumor cells it is called ct-DNA [21]. The existence of cf-DNA was first described by Madel and Metais in 1948 [23], but proper clinical attention was only given decades later when Leon $e t$ al. observed that cancer patients have a higher concentration of ccf-pDNA in serum or plasma when compared with healthy individuals [24]. Many evidences show that ccf-pDNA in these patients have genetic and epigenetic characteristics that are similar to the tumor, indicating that it actually originates from tumor tissue [25]. Therefore, ccf-pDNA could serve as a rapid and sensitive biomarker that can be obtained in a minimally invasive way for the evaluation of the diagnosis and prognosis of many different kinds of tumor [26].

Nowadays, it is known that cf-DNA, and more specifically ct-DNA, may be present in many biological fluids, such as blood (serum and plasma), urine and spittle. It is likely that cf-DNA release in the liquid biological matrix involves multiple processes that are dependent on the pathology or physiological state. It is also known that ccf-pDNAs originate from cell necrosis or apoptosis. Apoptosis, programmed cell death, is a normal physiological process whereas necrosis is a type of cell death that usually occur under pathological conditions that include neoplasias [27]. In healthy individuals, the main source of ccf-pDNA is the cells undergoing apoptosis, which release uniformly truncated fragments of DNA (185-200 base pair [bp] approximately). However, in malignant cells the main source of ccf-pDNA is necrosis, which generates larger fragments due to the incomplete and random digestion of genomic DNA [25]. Hence, the presence of larger fragments of ccf-pDNA in serum or plasma of an individual indicates the presence of some sort of pathology.

In order to analyze the size of the ccf-pDNA fragments in the plasma or serum of an individual, many studies have used the analysis of ALU sequences of repetitive DNA, classified as short interspersed elements, which encompasses $10 \%$ of the human genome [27,28]. The detection of fragments of ALU repetitive elements of 115 and $247 \mathrm{bp}$ is performed by quantitative PCR. Upon using different primer sets, the fragments of ALU repeats of $247 \mathrm{bp}$ (indication of necrotic DNA) or $115 \mathrm{bp}$ (indication of necrotic or apoptotic DNA) can be detected and quantified. With this information in hand, an integrity index for ccf-pDNA can be calculated. Khani et al. and Delgado et al. $[29,30]$ used this technique and showed that patients with community-acquired pneumonia presented with a concentration and integrity of plasma ccf-pDNA significantly higher than in healthy individuals or in those with hepato-pancreato-biliary cancer. A higher concentration of ccf-pDNA with a higher integrity rate are also related to the presence of lung cancer [31]. In this case, according to the authors, the higher the concentration of ccf-pDNA and integrity rate, the worse the prognosis for the patient. Zhang et al. used the same approach, and they revealed that higher concentrations of $219 \mathrm{bp}$ ALU fragments are associated with the presence of ovarian cancer [32].

Today, the Z-scan technique has been used in oncology. Fresh samples of tumor and healthy ovary tissues showed optical differences when measured by this technique [33]. An analysis of benign and malignant oral tumors revealed that the optical attenuation of the tumor samples was higher, and the nonlinear refraction index of the samples diverged in signal strength [34]. A study of clinical parameters in children with malignant solid tumors and/or 
Table 1. Summary of the main published articles on the study of Z-scan in the diagnosis of diseases.

\begin{tabular}{|c|c|c|c|}
\hline Study (year) & Journal & Samples & Z-scan use \\
\hline Gómes et al. (2010) & Chemistry and Physics of Lipids & Blood & $\begin{array}{l}\text { To quantify total cholesterol, triglycerides, protein, albumin } \\
\text { and glucose concentration }\end{array}$ \\
\hline Alves et al. (2008) & Chemistry and Physics of Lipids & Blood & To evaluate human lipoprotein oxidative stress \\
\hline Dhinna et al. (2008) & Laser Physics & Blood & To evaluate glucose levels \\
\hline Alves et al. (2014) & Liquid Crystals & Blood & Lyotropic-like low-density human lipoproteins \\
\hline Salman et al. (2016) & Optik & $\begin{array}{l}\text { Normal and cancerous } \\
\text { ovarian cells }\end{array}$ & To discriminate between normal and cancerous ovarian cells \\
\hline Salman et al. (2016) & Photodiagnosis and Photodynamic Therapy & $\begin{array}{l}\text { Normal and cancerous oral } \\
\text { tissue }\end{array}$ & To discriminate between benign and malignant oral tissue \\
\hline $\begin{array}{l}\text { do Nascimento } \\
\text { et al. (2019) }\end{array}$ & Photodiagnosis and Photodynamic Therapy & Blood & To detect cell-free circulating DNA \\
\hline
\end{tabular}

leukemia who were supplemented with selenium showed that the Z-scan method is much more sensitive to small variations in blood samples than the current techniques used in medical laboratories [15]. The use of this technique also enabled the determination of differences in ccf-pDNA between oncologic patients and healthy individuals as well as the verification of differences in the concentration of cf-DNA in plasma and urine of patients with bladder cancer undergoing chemotherapy [34]. Another example is the difference of sample signals of Z-scan method from a patients with breast cancer and leukemia after the treatment, the refractive index of urine DNA increases for 45 days, this result was compared with spectrophotometry technique and it was similar in both methods.

According to what was described above, it can be observed that the applicability of the Z-scan method goes beyond the determination of ccf-pDNA concentrations. It also seems to show molecular differences in DNA samples from patients and healthy individuals. The applicability can be added to the existing molecular methods for the development of new biomarkers and for the choice of therapeutic targets. We believe that the Z-scan method may soon be used in the diagnostic routine as a supporting method or, even as a primary choice, for diagnosis made by liquid biopsy samples.

There are no studies that compare, for the same analyte and for the same purpose, the Z-scan method with other methods, that can determine these analytes. Our group compared the Z-scan method with spectrophotometry in the detection of plasmatic DNA and this article was mentioned in this review [35]. The aim of this review is to show the Z-scan method as an alternative in determining, not to show if it is better or worse than other tests.

Table 1 shows the main studies that evaluated the potential use of the Z-scan technique for measurements in different biological samples.

\section{Conclusion \& future perspective}

This review showed that Z-scan technology can be a viable choice for diagnosis in cancer patients by determination of ccf-pDNA concentrations. This technique proves to be effective and minimally invasive, this being its main advantage over the other techniques currently used. It is possible to suggest that the improvement of the use of this technique be used in large scale in the coming years and help to supplement the diagnostic and prognostic techniques in oncology. However, considering the determination of an analyte, it is necessary to review physical rearrangement to applying the $\mathrm{Z}$-scan method. For this reason, studies that compare the Z-scan with other methods for the same analyte are required.

\section{Executive summary}

- Z-scan technology can be a viable choice for diagnosis in cancer patients.

- This technique has advantages over the other techniques currently used.

- Z-scan method goes beyond the determination of circulating cell-free DNA concentrations.

Author contributions

FLA Fonseca: formal analysis, data curation, writing the main manuscript, preparation of the figures, review and editing. BCA Alves and GL da Veiga: data curation, writing, review and editing. SIPMN Alves: data curation, formal analysis, preparation of the figures, review and editing. All authors reviewed the manuscript. 
Financial \& competing interest disclosure

The authors have no relevant affiliations or financial involvement with any organization or entity with a financial interest in or financial conflict with the subject matter or materials discussed in the manuscript. This includes employment, consultancies, honoraria, stock ownership or options, expert testimony, grants or patents received or pending, or royalties.

No writing assistance was utilized in the production of this manuscript.

Open access

This work is licensed under the Attribution-NonCommercial-NoDerivatives 4.0 Unported License. To view a copy of this license, visit http://creativecommons.org/licenses/by-nc-nd/4.0/

\section{References}

1. Califf RM. Biomarker definitions and their applications. Exp. Biol. Med. (Maywood) 243(3), 213-221 (2018).

2. FDA-NIH Biomarker Working Group. BEST (biomarkers, endpoints, and other tools) resource (2016). www.ncbi.nlm.nih.gov/books/NBK326791/

3. Aronson JK, Ferner RE. Biomarkers - a general review. Curr. Protoc. Pharmacol. 76, 9.23.21-9.23.17 (2017).

4. Sasseville VG, Mansfield KG, Brees DJ. Safety biomarkers in preclinical development: translational potential. Vet. Pathol. 51(1), 281-291 (2014).

5. Carr DF, Pirmohamed M. Biomarkers of adverse drug reactions. Exp. Biol. Med. (Maywood) 243(3), 291-299 (2018).

6. Puntmann VO. How-to guide on biomarkers: biomarker definitions, validation and applications with examples from cardiovascular disease. Postgrad. Med. J. 85(1008), 538-545 (2009).

7. Pepe MS, Thompson ML. Combining diagnostic test results to increase accuracy. Biostatistics 1(2), 123-140 (2000).

8. Liu D, Zhou XH. Covariate adjustment in estimating the area under ROC curve with partially missing gold standard. Biometrics 69(1), 91-100 (2013).

9. Tardif JC, Heinonen T, Orloff D, Libby P. Vascular biomarkers and surrogates in cardiovascular disease. Circulation 113(25), 2936-2942 (2006).

10. Sheik-Bahae M, Said AA, Van Stryland EW. High-sensitivity, single-beam n(2) measurements. Opt. Lett. 14(17), 955-957 (1989).

11. Sheik-Bahae M, Said AA, Wei T, Hagan DJ, Van Stryland EW. Sensitive measurement of optical nonlinearities using a single beam. IEEE J. Quantum Electron. 26(4), 760-769 (1990).

12. Gómez SL, Monteiro AM, Rabbani SR et al. Cu and Fe metallic ions-mediated oxidation of low-density lipoproteins studied by NMR, TEM and Z-scan technique. Chem. Phys. Lipids 163(6), 545-551 (2010).

13. Alves S, Monteiro AM, Gidlund MA, Figueiredo Neto AM. Thermal-lens effect of native and oxidized lipoprotein solutions investigated by the Z-Scan technique. Int. J. Atheroscler. 3(1), 33-38 (2008).

14. Dhinaa AN, Nooraldeen AY, Murali K et al. Z-scan technique as a tool for the measurement of blood glucose. Laser Phys. 18, 1212-1216 (2008).

15. Alves S, Neto Figueiredo AM. Advances in the non-linear optical investigation of lyotropic-like low-density human lipoproteins in the native and oxidised states. Liq. Cryst. 41(3), 465-470 (2014).

16. Monteiro AM, Jardini MA, Alves S et al. Cardiovascular disease parameters in periodontitis. J. Periodontol. 80(3), 378-388 (2009).

17. Alves S, Cuppo FLS, Figueiredo Neto AM. Determination of the nonlinear refractive index of lyotropic mixtures with and without ferrofluid doping: a time-resolved Z-scan experiment in millisecond time scales. J. Opt. Soc. Am. B 23(1), 67-74 (2006).

18. Alves S, Bourdon A, Figueiredo Neto AM. Generalization of the thermal lens model formalism to account for thermodiffusion in a single-beam Z-scan experiment: determination of the Soret coefficient. J. Opt. Soc. Am. B 20(4), 713-718 (2003).

19. Alix-Panabières C, Pantel K. Clinical Applications of circulating tumor cells and circulating tumor DNA as liquid biopsy. Cancer Discov. 6(5), 479-491 (2016).

20. Pantel K, Alix-Panabières C. Liquid biopsy and minimal residual disease - latest advances and implications for cure. Nat. Rev. Clin. Oncol. 16(7), 409-424 (2019).

21. Chu D, Park BH. Liquid biopsy: unlocking the potentials of cell-free DNA. Virchows Arch. 471(2), 147-154 (2017).

22. Gerlinger $\mathrm{M}$, Rowan $\mathrm{AJ}$, Horswell $\mathrm{S}$ et al. Intratumor heterogeneity and branched evolution revealed by multiregion sequencing. $N$. Engl. J. Med. 366(10), 883-892 (2012).

23. Mandel P, Metais P. Les acides nucléiques du plasma sanguin chez l'homme. C. R. Seances Soc. Biol. Fil. 142(3-4), 241-243 (1948).

24. Leon SA, Shapiro B, Sklaroff DM, Yaros MJ. Free DNA in the serum of cancer patients and the effect of therapy. Cancer Res. 37(3), 646-650 (1977).

25. Fawzy A, Sweify KM, El-Fayoumy HM, Nofal N. Quantitative analysis of plasma cell-free DNA and its DNA integrity in patients with metastatic prostate cancer using ALU sequence. J. Egypt Natl Canc. Inst. 28(4), 235-242 (2016). 
26. Francis G, Stein S. Circulating cell-free tumour DNA in the management of cancer. Int. J. Mol. Sci. 16(6), 14122-14142 (2015).

27. Utomo WK, Janmaat VT, Verhaar AP et al. DNA integrity as biomarker in pancreatic cyst fluid. Am. J. Cancer Res. 6(8), 1837-1841 (2016).

28. Hwu HR, Roberts JW, Davidson EH, Britten RJ. Insertion and/or deletion of many repeated DNA sequences in human and higher ape evolution. Proc. Natl Acad. Sci. USA 83(11), 3875-3879 (1986).

29. Khani M, Hosseini J, Mirfakhraie R, Habibi M, Azargashb E, Pouresmaeili F. The value of the plasma circulating cell-free DNA concentration and integrity index as a clinical tool for prostate cancer diagnosis: a prospective case-control cohort study in an Iranian population. Cancer Manag. Res. 11, 4549-4556 (2019).

30. Delgado PO, Alves BC, de Sousa Gehrke F et al. Characterization of cell-free circulating DNA in plasma in patients with prostate cancer. Tumor Biol. 34(2), 983-986 (2013).

31. Chudasama D, Katopodis $\mathrm{P}$, Stone $\mathrm{N}$ et al. Liquid biopsies in lung cancer: four emerging technologies and potential clinical applications. Cancers (Basel) 11(3), 331 (2019).

32. Zhang R, Pu W, Zhang S et al. Clinical value of ALU concentration and integrity index for the early diagnosis of ovarian cancer: a retrospective cohort trial. PLoS ONE 13(2), e0191756 (2018).

33. Salman M, Hosein MAM, Mohammad N. Nonlinear optical investigation of normal ovarian cells of animal and cancerous ovarian cells of human in-vitro. Optik 127, 3867-3870 (2016).

34. Salman M, Hossein MA, Kamran KS, Shayan M. Optical discrimination of benign and malignant oral tissue using Z-scan technique. Photodiagnosis Photodyn. Ther. 16, 54-59 (2016).

35. do Nascimento Alves SIPM, Lavalhegas Hallack M, Moreira Perez M, da Costa Aguiar Alves B, da Silva LH, Afonso Fonseca FL. Application of the Z-scan technique for the detection of CFCDNA (cell-free circulating DNA) and urine DNA (uDNA) in patients with bladder cancer. Photodiagnosis Photodyn. Ther. 26, 131-133 (2019). 